\title{
ESTIMACIÓN DE LA POBLACIÓN DE PERROS CALLEJEROS EN EL DISTRITO DE LOS OLIVOS, LIMA, PERÚ
}

\author{
Estimated Population of Stray Dogs in the District of Los Olivos, \\ Lima, Peru
}

Yaliní Ochoa A. ${ }^{1,3}$, Néstor Falcón P. ${ }^{1,4}$, José Zuazo R. ${ }^{1}$, Beder Guevara P. ${ }^{2}$

\section{Resumen}

El objetivo del estudio fue estimar y caracterizar la población de perros callejeros en el distrito de Los Olivos durante el periodo noviembre y diciembre 2012. Para ello se seleccionaron 8 de las 34 zonas que divide al distrito en el plano municipal. La selección de las zonas fue de forma aleatoria y para el conteo y cálculos de la población de perros se utilizaron los criterios expuestos en la guía «Censando poblaciones de perros callejeros: guía metodológica» de la World Society for the Protection of Animals. Se realizaron tres muestreos consecutivos en horarios diurno y nocturno en cada zona. Se utilizó la media de las tres mediciones para realizar los cálculos estadísticos. El estudio reportó un promedio de 332 perros callejeros en horario diurno y de 217 en horario nocturno. Los animales machos y los de tamaño grande se observaron en mayor número. La mayoría de perros poseía un índice de condición corporal bajo. La estimación del número de perros callejeros en el distrito fue de $1411 \pm 643$ en horario diurno y de $922 \pm 497$ en horario nocturno. Se espera que los resultados aporten información de línea base al programa de control poblacional canino que viene trabajando la Municipalidad Distrital de Los Olivos.

Palabras clave: perros callejeros, Los Olivos, zoonosis, mordeduras

\section{Abstract}

The aim of this study was to estimate and characterize the stray dog population in Los Olivos district, Lima, during the period November - December 2012. Eight of the 34 areas that comprise the district according the map of Los Olivos Municipality were selected. The selection of the areas was at random; dog population counting and

\footnotetext{
${ }^{1}$ Facultad de Medicina Veterinaria y Zootecnia, Universidad Peruana Cayetano Heredia, Lima

${ }^{2}$ Municipalidad Distrital de Los Olivos, Lima

${ }^{3}$ E-mail: yalinimoa15@hotmail.com

${ }^{4}$ E-mail:nfalconperez@gmail.com
}

Recibido: 1 de noviembre de 2013

Aceptado para publicación: 8 de abril de 2013 
calculations were based on the criteria outlined in the guide of the World Society for the Protection of Animals entitled «Recensusing roaming dog populations: guidelines on methodology». Three consecutive sampling were performed at daytime and evening hours in each area. The average of the three measurements was used for statistical calculations. There was an average of 332 stray dogs at daytime and 217 stray dogs at evening; males and bigger dogs were observed in higher number. Most of the dogs showed low body condition score. The estimated number of stray dogs in the district was $1411 \pm 643$ at daytime and $922 \pm 497$ at night. Results are expected to contribute with the dog control population programme that is being implemented by the Municipality of Los Olivos.

Key words: stray dogs, Los Olivos, zoonosis, bites

\section{INTRODUCCIÓN}

Se define como perro callejero aquel que ha sido abandonado o que aun teniendo dueño deambula libremente por las áreas públicas sin control directo (Brusoni et al., 2007; Guerra et al., 2007; WSPA, 2007; OIE, 2010). Estos animales son echados de las casas para que busquen su alimento entre la basura y los desperdicios en las calles y mercados o son abandonados cuando los dueños no se encuentran en capacidad de mantenerlos (Brusoni et al., 2007; Romero, 2008). De hecho, en muchos países, la mayoría de perros que se definen como callejeros tienen dueño, pero se les permite vagar por sitios públicos durante parte o todo el día. Esta es una manifestación de una serie de factores socioeconómicos y culturales que tiene su origen en la insuficiente educación ciudadana y en una deficiente legislación sobre el impacto de la población canina en el medio ambiente y la salud pública (Álvarez y Domínguez, 2001; Ibarra et al., 2006a).

Ante la incapacidad de obtener hogares para albergar a los perros callejeros, estos aprovechan estructuras urbanas abiertas y abandonadas como lugares de refugio temporal, fenómeno que se observa en la mayoría de las ciudades de América Latina (Molina et al., 2006). Estos animales representan un problema social debido a que se convierten en factores contaminantes del ambiente (parques y jardines públicos), representan un riesgo para la transmisión de enfermedades zoonóticas, producen disturbios sonoros por ladridos excesivos, peleas entre ellos e incluso agresiones a las personas (Güttler, 2005; ICAM, 2007).

La población de perros callejeros debe ser controlada mediante el fomento de la tenencia responsable a fin de disminuir los riesgos que ocasionan (OIE, 2010; Zunino et al., 2000). Para ello se hace necesario educar a la población, especialmente a los más jóvenes quienes suelen ser los más interesados en estos temas y aprovechando las escuelas como lugares para transmitir la información (Rondón, 2011). Además, en el caso de la ciudad de Lima, Perú, se requiere estimar el tamaño de la población de estos perros a fin de contar con una línea base para los trabajos de tenencia responsable de animales de compañía que las municipalidades deben realizar en el marco de la Ley N. ${ }^{\circ} 27596$, que regula el Régimen Jurídico de Canes.

No se dispone de estudios específicos sobre las características de la población de perros callejeros en la ciudad de Lima. Por ello, el objetivo del estudio fue estimar y caracterizar esta población de perros del distrito de Los Olivos. 


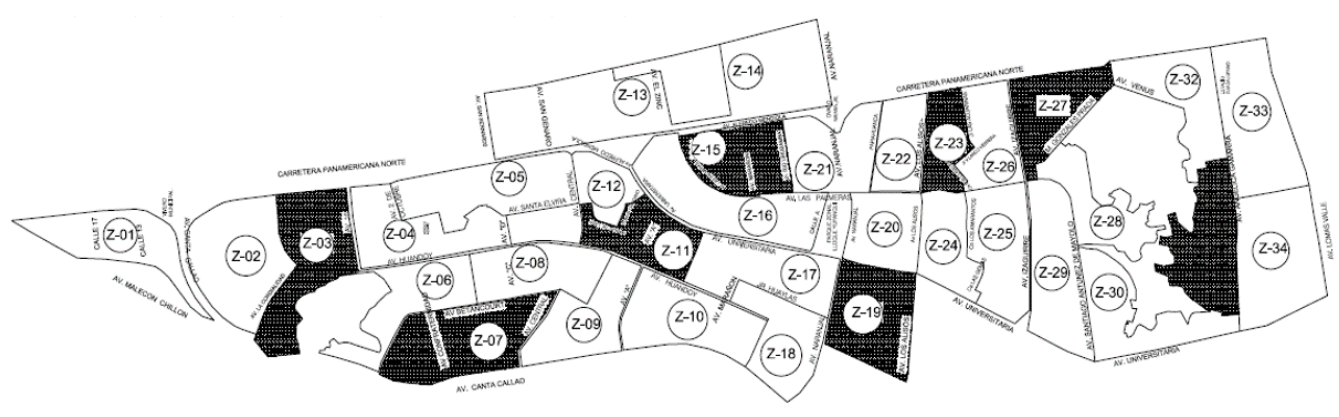

Figura 1. Mapa del Distrito de Los Olivos, mostrando las zonas seleccionadas para el muestreo

\section{Materiales y Métodos}

El estudio se realizó en el distrito de los Olivos, localizado en la zona norte de Lima Metropolitana. El distrito tiene una extensión de $17.3 \mathrm{~km}^{2}$ y una población aproximada de 318 mil habitantes (INEI, 2007). El tipo de investigación correspondió a un estudio observacional, transversal y descriptivo.

Según la metodología descrita por la WSPA (2007), se dispuso de un mapa del distrito con divisiones correspondientes a los establecimientos de expendios de alimentos. El mapa estaba dividido en 34 zonas y la selección de los zonas se realizó mediante un muestreo probabilístico (sistemático) buscando asegurar la aleatorización de la muestra (Fig. 1). Básicamente se realizaron los siguientes procedimientos:

- El mapa estaba dividido en 34 zonas, las que se enumeraron consecutivamente del 1 al 34 .

- Se incluyó en el censo al 25\% de las zonas. Para este fin, se escogió un número al azar entre 1 y 4 , siendo este el 3 .

- Siguiendo los criterios de un muestreo sistemático, la zona 3 fue la primera considerada en la muestra. Se sumó a este dígito el número 4 en forma consecutiva, quedando seleccionados de esta forma las zonas $3,7,11,15,19,23,27$ y 31 , representando una muestra aleatoria de las zonas del distrito.

Para el censado de los canes, se trabajó una zona por día, en los horarios de 05:00 a 07:00 y de 22:00 a 24:00 horas. Cada zona se recorrió dos veces durante el día (uno para el muestreo en horario diurno y el otro para el horario nocturno). Los recorridos se hicieron a pie y con una duración aproximada de 2 horas con la finalidad de obtener información de la cantidad de perros que se encontraban deambulando en el momento de los muestreos. A fin de mejorar la confiabilidad de la estimación de la población de canes callejeros en una determinada zona, se realizaron tres mediciones en tres días consecutivos en cada uno de los horarios predefinidos, transitando por todas las calles de las zonas seleccionadas tratando en lo posible de repetir la misma secuencia de recorrido. Con esta información, se obtuvo el número promedio de perros callejeros, el cual fue utilizado para la estimación de la población total en el distrito. La municipalidad apoyó el estudio proporcionando una moto, un personal para su manejo, seguridad y mapas guías detallando las zonas seleccionadas. 
Cuadro 1. Número de perros callejeros contabilizados en dos horarios con tres muestreos consecutivos en el distrito de los Olivos, Lima, Perú (noviembre-diciembre, 2012)

\begin{tabular}{ccccccccc}
\hline \multirow{2}{*}{ Zona } & \multicolumn{3}{c}{ Conteo entre 22:00 a 24:00 horas } & \multicolumn{3}{c}{ Conteos entre 05:00 a 07:00 horas } \\
\cline { 2 - 8 } & 1 & 2 & 3 & Promedio & 1 & 2 & 3 & Promedio \\
\hline 3 & 4 & 17 & 18 & 13 & 10 & 37 & 33 & 27 \\
7 & 54 & 65 & 53 & 58 & 49 & 82 & 98 & 77 \\
11 & 40 & 47 & 37 & 42 & 75 & 43 & 55 & 58 \\
15 & 18 & 17 & 11 & 15 & 37 & 18 & 35 & 30 \\
19 & 6 & 13 & 9 & 9 & 11 & 10 & 15 & 12 \\
23 & 34 & 26 & 30 & 30 & 50 & 28 & 51 & 43 \\
27 & 10 & 5 & 10 & 8 & 13 & 7 & 22 & 14 \\
31 & 35 & 39 & 53 & 42 & 71 & 63 & 78 & 71 \\
\hline Total & 201 & 229 & 221 & 217 & 316 & 288 & 387 & $332^{1}$ \\
\hline
\end{tabular}

Se trabajó una zona por semana. Se contaron todos los perros que se encontraban dentro de los límites de cada zona, poniendo énfasis en la observación de posibles escondites (debajo de automóviles, drenajes). Se tomaron datos de sexo (macho, hembra, indeterminado), tamaño (pequeño, mediano, grande) y estado de bienestar (presencia de lesiones en piel, cojeras, condición corporal), siguiendo los criterios de las Guías para la Evaluación Nutricional de Perros y Gatos de la Asociación Americana Hospitalaria de Animales (Baldwin et al., 2010). Adicionalmente, se tomaron fotografías como herramientas de apoyo al conteo de perros, cuando las condiciones no eran propicias para el conteo individual.

La estimación del total de perros callejeros se hizo siguiendo los criterios de la guía metodológica del WSPA (WSPA, 2007). Los resultados se presentan como media estimada \pm intervalo de confianza. La diferencia de proporciones entre las variables en estudio dentro de cada muestreo y entre momentos de muestreo se evaluó mediante la prueba de Chi cuadrado.

\section{Resultados}

El estudio reportó un promedio de 332 y 217 perros callejeros en los conteos realizados en horarios diurno y nocturno, respectivamente (Cuadro 1). El menor número promedio de perros se presentó en las zonas 3, 15,19 y 27 en ambos horarios, en tanto que el mayor número fue observado en las zonas $7,11$ y 31 en ambos horarios ( $\mathrm{p}<0.05)$. Asimismo, se observó mayor cantidad de perros machos y perros grandes $(\mathrm{p}<0.05)$ en ambos horarios en relación a hembras y perros de otros tamaños (Cuadro 2).

Entre los indicadores de bienestar se encontró que la principal característica afectada fue el Índice de Condición Corporal. Por otro lado, no se encontraron diferencias entre las proporciones de animales afectados en los tres indicadores por efecto del horario del conteo (Cuadro 3).

En base al número estimado de perros callejeros contabilizados se estimó que existían $1411 \pm 643$ perros en las calles en hora- 
Cuadro 2. Promedio de perros callejeros según procedencia, sexo y tamaño en el distrito de Los Olivos, Lima (noviembre-diciembre de 2012)

\begin{tabular}{|c|c|c|c|c|c|}
\hline \multirow{2}{*}{ Variable } & \multirow{2}{*}{ Estrato } & \multicolumn{2}{|c|}{$\begin{array}{c}\text { Entre 22:00 a 24:00 horas } \\
(\mathrm{n}=217)\end{array}$} & \multicolumn{2}{|c|}{$\begin{array}{c}\text { Entre 05:00 a 07:00 horas } \\
(\mathrm{n}=332)\end{array}$} \\
\hline & & $\mathrm{n}$ & $\%$ & $\mathrm{n}$ & $\%$ \\
\hline \multirow{8}{*}{ Zona } & 27 & 8 & $3.7^{\mathrm{a}}$ & 14 & $4.2^{\mathrm{a}}$ \\
\hline & 19 & 9 & $4.1^{\mathrm{a}}$ & 12 & $3.6^{\mathrm{a}}$ \\
\hline & 3 & 15 & $6.0^{\mathrm{a}, \mathrm{b}}$ & 27 & $8.1^{\mathrm{ab}}$ \\
\hline & 5 & 15 & $6.9^{\mathrm{a}, \mathrm{b}}$ & 30 & $9.0^{\mathrm{ab}}$ \\
\hline & 23 & 30 & $13.8^{\mathrm{b}, \mathrm{c}}$ & 43 & $13.0^{\mathrm{b}, \mathrm{c}}$ \\
\hline & 11 & 42 & $19.4^{\mathrm{c}, \mathrm{d}}$ & 58 & $17.5^{\mathrm{c}, \mathrm{d}}$ \\
\hline & 31 & 42 & $19.4^{\mathrm{c}, \mathrm{d}}$ & 71 & $21.4^{\mathrm{d}}$ \\
\hline & 7 & 58 & $26.7^{\mathrm{d}}$ & 77 & $23.2^{\mathrm{d}}$ \\
\hline \multirow{3}{*}{ Sexo } & Macho & 128 & $59.0^{\mathrm{a}}$ & 199 & $59.9^{\mathrm{a}}$ \\
\hline & Hembra & 32 & $14.7^{\mathrm{c}}$ & 51 & $15.4^{\mathrm{c}}$ \\
\hline & Indeterminado ${ }^{1}$ & 57 & $26.3^{\mathrm{b}}$ & 82 & $24.7^{\mathrm{b}}$ \\
\hline \multirow{3}{*}{ Tamaño } & Pequeño & 32 & $14.7 \mathrm{a}$ & 67 & $20.2 \mathrm{a}$ \\
\hline & Mediano & 83 & $38.2^{\mathrm{b}}$ & 113 & $34.0^{\mathrm{b}}$ \\
\hline & Grande & 102 & $47.0^{\mathrm{b}}$ & 152 & $45.8^{\mathrm{c}}$ \\
\hline
\end{tabular}

a,b,c Superíndices diferentes indican diferencia significativa dentro de variables y dentro de horarios de conteo $(p<0.05)$

${ }^{1}$ No se pudo determinar el sexo

Cuadro 3. Frecuencia de perros callejeros con características que afectan su bienestar en el distrito de los Olivos, Lima (noviembre-diciembre de 2012)

\begin{tabular}{lcccc}
\hline \multirow{2}{*}{ Parámetro } & \multicolumn{2}{c}{$\begin{array}{c}\text { Entre 22:00 a 24:00 horas } \\
(\mathrm{n}=217)\end{array}$} & \multicolumn{2}{c}{$\begin{array}{c}\text { Entre 05:00 a 07:00 horas } \\
(\mathrm{n}=332)\end{array}$} \\
\cline { 2 - 5 } & $\mathrm{n}$ & $\%$ & $\mathrm{n}$ & $\%$ \\
\hline Lesiones en piel & 46 & $21.2^{\mathrm{a}}$ & 73 & $22.0^{\mathrm{a}}$ \\
Cojera & 13 & $6.0^{\mathrm{a}}$ & 26 & $7.8^{\mathrm{a}}$ \\
Baja condición corporal & 124 & $57.1^{\mathrm{a}}$ & 207 & $62.3^{\mathrm{a}}$ \\
\hline
\end{tabular}

${ }^{a}$ Superíndices similares indican que no hubo diferencias estadísticas dentro de variables por efecto del horario de conteo 
rio diurno y $922 \pm 497$ en horario nocturno. No se observaron diferencias entre el número de perros callejeros entre los horarios de muestreo.

\section{Discusión}

Aunque no existe diferencia significativa en la estimación de perros según el horario de muestreo, se observa que existe una tendencia a observar una mayor cantidad de perros callejeros en el horario diurno. Ello se explicaría por el hecho de que existe una costumbre de dejar en libertad a los canes en el día para que defequen en las calles y busquen comida, así como, en algunos casos, para que permanezcan en los exteriores cumpliendo función de guardianes en algún local. Esta información se obtuvo durante las entrevistas a las personas del distrito. Si bien es cierto esto no estuvo considerado en la metodología, las entrevistas se realizaron de manera informal (no hubo un documento de encuesta) con la finalidad de encontrar información que explique el manejo de los perros por parte de la población. Por otro lado, el menor número de animales en la noche probablemente sea debido a que una parte de ellos ingresan a sus lugares de procedencia.

La permanencia de animales callejeros representa un potencial factor de riesgo para la salud de las personas porque estos animales, especialmente aquellos que no tienen dueño, no recibirían atención veterinaria y podrían actuar como reservorios de enfermedades (Ej. endoparásitos como Toxoca canis y ectoparásitos como las pulgas que pueden transmitir Dipylidium caninum), como fuente de contaminación ambiental, así como ocasionar lesiones por mordeduras (Ibarra et al., 2006b; Álvarez y Domínguez, 2001).

La mayor parte de los perros callejeros mostraban una baja condición corporal, probablemente a consecuencia de una inadecuada alimentación, especialmente en aquellos sin dueño. Estos mismos estarían más pro- pensos a sufrir problemas de piel, como sarna sarcóptica y demodéctica, o por agentes bacterianos y micóticos. Estas afecciones se traducen en una deficiente salud, que en caso de tener un carácter endémico representaría un foco de infección para otros animales que son paseados en parques o calles por sus propietarios (Guerra et al., 2007; Güttler, 2005).

Durante el conteo de perros callejeros se encontraron valores extremos, de 8 a 77 perros. Esto podría deberse a que en las zonas seleccionadas había diversos estratos, tales como zona media baja, zona media y zona media alta (INEI, 2009). En las zonas medias bajas se contabilizó mayor cantidad de estos perros. Asimismo, allí se encontraba la mayoría de animales con baja condición corporal y con problemas de piel y cojeras. Es probable que en estas zonas se brinden menores cuidados a los perros, en contraste con las zonas socioeconómicas medias y altas, donde se encontró menor cantidad de perros callejeros. En estas zonas se ha observado que la permanencia de los perros con dueños en la calle suele ser temporal. Los animales permanecen un tiempo limitado en la calle para que orinen, defequen o simplemente paseen, después del cual son nuevamente retornados a las casas.

Durante el conteo se observó mayor cantidad de perros machos que hembras, siendo estos resultados semejantes a los encontrados en Chile (Morales et al., 2009; Ibarra et al., 2006b), aunque estos resultados se obtuvieron a base de encuestas. Sin embargo, no se pudo determinar el sexo en una gran cantidad de animales debido a la posición en la que se encontraban (decúbito ventral), el pelaje abundante o la distancia del lugar en que se encontraban al sitio de observación.

El estudio también encontró mayor cantidad de perros grandes. Esto fue similar a lo encontrado en Chile (Ibarra et al., 2006b) y se le atribuyó a las preferencias de los propietarios para usar estos animales como guardianes. Se encontraron animales en la puerta de las casas durmiendo o cuidando, algunos 
lo hacían en casas o camas implementadas artesanalmente, en donde los perros pasaban la noche; no obstante, no se encontraron crías en esta situación. Estos resultados fueron similares a los encontrados en un estudio de estimación de la población de perros vagabundos en la Ciudad de Buenos Aires, donde se menciona que la razón de la ausencia de crías se debería a su mayor susceptibilidad a enfermedades infecciosas virales (parvovirus, distemper), disminuyendo sus posibilidades de supervivencia: asimismo, tienen más posibilidades de ser recogidos y llevados a un hogar como un acto de compasión (Molina et al., 2006).

En el caso del Distrito de Los Olivos, se ha promulgado una ordenanza municipal (Nro. 184-CDLO, aprobada en 2004), basada en la Ley $N^{\circ} 27596$. Dentro de ese marco se han realizado actividades de registro de perros, campañas de esterilización y desparasitación, y actividades de sociabilización de los alcances de la Ordenanza Municipal a través de charlas en colegios y ambientes públicos. En la parte correspondiente a los perros callejeros, la ordenanza establece que estos deben ser capturados por las autoridades municipales (inspector sereno o personal técnico que apoye el grupo de trabajo de salubridad y saneamiento ambiental de la municipalidad) y deben ser puestos a disposición de la autoridad del sector salud, albergues para animales o establecimientos autorizados para tal fin, en tanto se implemente el albergue municipal canino. La Municipalidad de Los Olivos aún no dispone del albergue canino, facilitando la presencia de animales callejeros.

El presente estudio sienta la línea base a partir de la cual se puede evaluar el comportamiento de esta población una vez que se disponga la intervención municipal sobre los perros callejeros. Ello permitirá evaluar el efecto de la intervención o reformular las actividades que están obligadas a realizar, a fin de regular la presencia de estos canes en espacios públicos con el fin de prevenir potenciales problemas que pudieran afectar a la salud pública.

\section{Conclusiones}

- Se estima que existen $1411 \pm 643$ perros callejeros en horario diurno y de 922 \pm 497 en horario nocturno en el distrito de Los Olivos, Lima.

- El número de perros callejeros fue estadísticamente similar en los horarios nocturnos (22:00 a 24:00 horas) y diurnos (05:00 a 07:00 horas). Asimismo, se observó mayor cantidad de perros callejeros de sexo macho y de tamaño grande.

\section{Literatura Citada}

1. Álvarez E, Domínguez J. 2001. Programa para el control integral de la población canina. AMMVEPE 12(3): 83-91.

2. Baldwin K, Bartges J, Buffington T, Freeman L, Grabow M, Legred J, et al. 2010. Guías para la evaluación nutricional de perros y gatos de la Asociación Americana Hospitalaria de Animales (AAHA). J Am Anim Hosp Assoc 46(4). [Internet]. Disponible en: http:// www.aahanet.org/PublicDocuments/ NAG_Spanish_color.pdf

3. Brusoni C, Fernández Canigia J, Lara J, Dezzotti A. 2007. Tamaño y estructura de la población canina en San Martin de los Andes (Neuquén). Analecta Veterinaria 27(1): 11-23.

4. Guerra Y, Echagarrúa Y, Marin E, Mencho J, Marín A, Pascual T, et al. 2007. Factores que conllevan al abandono de perros en una región de Cuba. REDVET 8(12). [Internet] [01 junio 2012]. Disponible en: http://www.redalyc.org/articulo.oa?id=63681204

5. Güttler V. 2005. Análisis de algunas características de la población canina relacionadas con mordeduras e hidatidosis humana en la provincia de Valdivia. Tesis de Médico Veterinario. Chile: Universidad Austral de Chile. 60 p.

6. Ibarra L, Espínola F, Echeverría M. 2006a. Factores relacionados con la presencia de perros en las calles de la ciu- 
dad de Santiago, Chile. Avances en Ciencias Veterinarias 21(1-2). [Internet]. Disponible en: http://www.avances veterinaria. uchile.cl/index.php/ACV/article/ viewFile/1384/1279

7. Ibarra L, Espínola F, Echeverría M. 2006b. Una prospección a la población de perros existentes en las calles de la ciudad de Santiago, Chile. Avances en Ciencias Veterinarias 21(1-2). [Internet]. Disponible en: http://www.avancesveterinaria.uchile.cl/index.php/ACV/ article/viewArticle/3953/3857

8. [ICAM] Coalición Internacional para el Manejo de Animales de Compañía. 2007. Guía para el manejo humanitario de poblaciones caninas. 24 p. [Internet]. Disponible en: http://www.icam-coalition. org/downloads/ Guia_Para_El_ Manejo_-Humanitario_de_Poblaciones_Caninas_Spanish.pdf

9. [INEI] Instituto Nacional de Estadística e Informática. 2007. Características de la población. Perú. 1: 72 p.

10. [INEI] Instituto Nacional de Estadística e Informática. 2009. Planos Estratifi-cados de Lima Metropolitana a nivel de manzanas. Lima: INEI. 123 p. [Internet]. Disponible en: http:// www.catalogo.uni.edu.pe/cgi-bin/koha/ opac-detail.pl?biblionumber $=50728$ \&shelfbrowse_itemnumber $=78788$

11. Ley No. 27596 Régimen jurídico de canes. Diario El Peruano. 2002. p.225240-225244.

12. Molina JL, Faigenbaum A, Castro JR, Gastrel H, Ruggia R. 2006. Estimación de la población de caninos vagabundos en la Ciudad de Buenos Aires, octubre-noviembre 2006. Instituto de zoonosis
Luis Pasteur Ministerio de Salud GCAB. 4 p. [Internet]. Disponible en: http://salud.ciee.flacso.org.ar/files/ flacso/pasteur/pdf/EstPobCanVag.pdf

13. Morales MA, Varas C, Ibarra $L$. 2009. Caracterización demográfica de la población de perros de Viña del Mar, Chile. Arch Med Vet 41: 89-95.

14. [OIE] World Organisation for Animal Health. 2010. Terrestrial Animal Health Code. Chap. 7.7. Stray dog population control. [Internet]. Disponible en: http:// www.oie.int/fileadmin/Home/eng/ Health_standards/tahc/2010/ en_chapitre_1.7.7.htm

15. Romero C. 2008. Tenencia responsable de mascotas caninas en la comuna de Florida. Región Metropolitana. Tesis de Médico Veterinario. Santiago de Chile: Universidad Iberoamericana de Ciencias y Tecnología. $40 \mathrm{p}$.

16. Rondón H. 2011. Endoparásitos gastrointestinales zoonóticos en mascotas y ambientes en contacto con escolares de nivel primaria en instituciones educativas de los distritos del cono norte de Lima Metropolitana. Tesis de Maestría. Lima: Universidad Peruana Cayetano Heredia. $77 \mathrm{p}$.

17. [WSPA] Sociedad Mundial de Protección Animal. 2007. Censando poblaciones de perros deambulantes: guía metodológica. Londres: WSPA. 21 p.

18. Zunino MG Defrancesco MU, Kuruc J, Schweigmann M, Wisnivesky MC, Jensen O. 2000. Contaminación por helmintos en espacios públicos de la provincia de Chubut. Argentina. Bol Chil Parasitol 55(3-4): 1-6. 